\title{
Quantitative characterization of the $x$-ray imaging capability of rotating modulation collimators with laser light
}

\author{
C. C. Gaither III, E. J. Schmahl, C. J. Crannell, B. R. Dennis, F. L. Lang, L. E. Orwig, \\ C. N. Hartman, and G. J. Hurford
}

\begin{abstract}
We developed a method for making quantitative characterizations of bi-grid rotating modulation collimators (RMC's) that are used in a Fourier transform x-ray imager. With appropriate choices of the collimator spacings, this technique can be implemented with a beam-expanded He-Ne laser to simulate the plane wave produced by a point source at infinity even though the RMC's are diffraction limited at the He-Ne wavelength of $632.8 \mathrm{~nm}$. The expanded beam passes through the grid pairs at a small angle with respect to their axis of rotation, and the modulated transmission through the grids as the RMC's rotate is detected with a photomultiplier tube. In addition to providing a quantitative characterization of the RMC's, the method also produces a measured point response function and provides an end-to-end check of the imaging system. We applied our method to the RMC's on the high-energy imaging device (HEIDI) balloon payload in its preflight configuration. We computed the harmonic ratios of the modulation time profile from the laser measurements and compared them with theoretical calculations, including the diffraction effects on irregular grids. Our results indicate the 25 -in. (64-cm) x-ray imaging optics on HEIDI are capable of achieving images near the theoretical limit and are not seriously compromised by imperfections in the grids. () 1996 Optical Society of America
\end{abstract}

Key words: Fourier transform imaging, imaging systems, laser, applications, optical testing, rotating modulation collimators, x-ray imagers.

\section{Introduction}

Recent results from data obtained with the Japanese Yohkoh satellite indicate that high spatial and temporal resolution hard x-ray and gamma-ray imaging

When this research was undertaken, C. C. Gaither III was a National Academy of Sciences/National Research Council Fellow in Code 682, Solar Physics Branch, NASA Goddard Space Flight Center, Greenbelt, Maryland 20771. He is now with the Laboratory of Cardiac Energetics, National Heart, Lung, and Blood Institute of the National Institutes of Health, Bethesda, Maryland 20892. E. J. Schmahl, C. J. Crannell, B. R. Dennis, F. L. Lang, L. E. Orwig are in Code 682, NASA Goddard Space Flight Center. E. J. Schmahl is also with the Department of Astronomy, University of Maryland, College Park, Maryland 20742; and F. L. Lang is also with the Department of Physics, The Catholic University of America, Washington, D.C. 20064. C. N. Hartman is in Code 735, Flight Data Systems Branch, NASA Goddard Space Flight Center. G. J. Hurford is with Solar Astronomy, California Institute of Technology, Pasadena, California 91103.

Received 23 October 1995; revised manuscript received 23 February 1996.

0003-6935/96/06714-13\$10.00/0

(C) 1996 Optical Society of America spectroscopy can provide definitive answers to fundamental questions concerning the evolution of solar flares. ${ }^{1-3}$ The high-energy solar imager (HESI) is currently being studied as a Fourier transform imager with finer spectral and spatial resolution and an energy response that extends into the gamma-ray domain. ${ }^{4}$ It builds on the technological heritage developed with the high-energy imaging device (HEIDI) and the high-resolution gamma-ray spectrometer balloon payloads. ${ }^{5,6}$

Hard $\mathrm{x}$ rays and gamma rays cannot be reflected or focused with lenses or mirrors. Even grazingincidence reflection, used effectively in both solar and celestial soft x-ray astronomy, is impossible in the photon energy domain above a few tens of kiloelectron volts. What is required is a variation on the pinhole camera consisting of transparent apertures in material opaque to these radiations. Although the Fourier transform technique as implemented for HESI provides an effective collecting area of only one fourth the total detector area, it is a feasible approach for solar flares and other high-flux phenomena. Simulations with software similar to that which would be used to analyze observations with HESI 


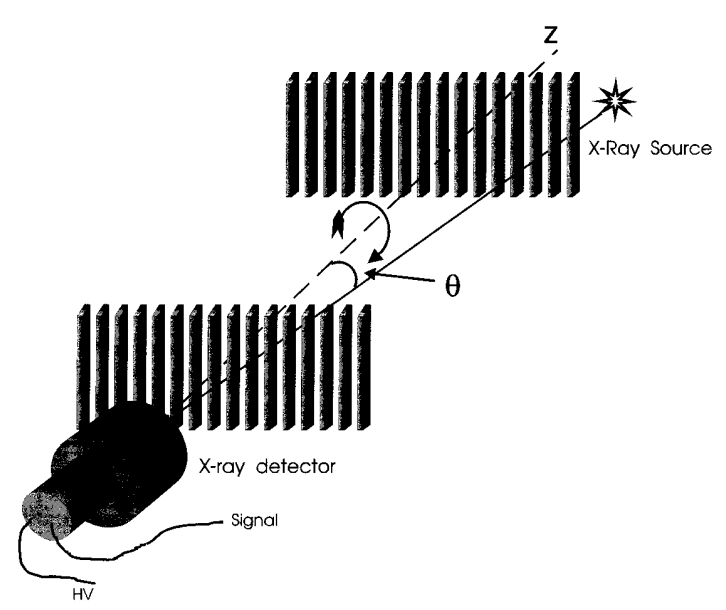

Fig. 1. Schematic of the RMC's in the HEIDI telescope. The grid pairs were coaligned, forming RMC's within the telescope assembly, which was designed to point within a small fraction of a degree (labeled $\theta$ ) of an x-ray source and rotated about the axis of symmetry (labeled Z). An x-ray detector was situated behind the rear grid of each RMC and measured the x-ray flux from the source as modulated by the rotating grid pair. The x-ray detector does not rotate with the grid pair. HV, high voltage.

have demonstrated that extended sources, with features such as magnetic arches with angular extents in the range from 2 arc sec to 2 arc min, could be imaged with HESI.

The Fourier transform imaging technique employed with HESI is implemented with a set of 12 rotation modulation collimators (RMC's). ${ }^{4}$ A single RMC consists of two uniform grids, with identical pitch, made from a high- $Z$ material such as tungsten, separated by a large distance compared with the slit width of the grids. The range of grid-pitch sizes is chosen so that the source sizes to which the RMC's are sensitive span the range of source sizes anticipated to be of interest for the high-energy emissions from solar flares. The grid pairs are coaligned, forming RMC's within the telescope assembly, which is pointed to within a small fraction of a degree of Sun center and rotated. An x-ray detector is situated behind the rear grid of each RMC and measures the $\mathrm{x}$-ray flux from the source as modulated with the rotating grid pair as shown in Fig. 1. The grids rotate around their axis of symmetry, labeled $\mathrm{Z}$ in Fig. 1. Note that this technique imposes no requirement with respect to position sensitivity on the x-ray detectors, which are required only to measure the counting rate as a function of time and photon energy. The RMC's modulate the x-ray flux observed with the detector by occulting and de-occulting alternately the source, providing that a symmetric x-ray source is not situated on the rotation axis of the collimators. If the x-ray source is located on the rotation axis, then no modulation of the incoming x-ray flux occurs. In a short time interval compared with a rotation period, the fundamental of the modulated signal from an individual RMC provides information on one spatial Fourier component of the source being observed. This information can be characterized as two symmetric points in the Fourier transform (UV) plane. In half a rotation $\left(180^{\circ}\right)$, the information from an individual RMC can be represented by a complete circle in the UV plane at a radius corresponding to the angular dimension determined by the grid pitch and the grid separation. Thus, in the time required for half a rotation, the 12 RMC's together provide all the information necessary to construct an image of the source covering the range of source sizes of interest.

We developed a method to characterize quantitatively the x-ray imaging capabilities of RMC's with visible light. In addition to providing a preflight calibration, this technique provides a quick and relatively simple way for determining whether the theoretical spatial resolution achievable with a given $\mathrm{RMC}$ has been compromised during integration into either a balloon or a satellite payload. RMC's, as with other shadow-casting x-ray imagers, are difficult to calibrate with $\mathrm{x}$ rays, $, 3,7,8$ particularly when they are designed for such fine angular resolution [ 2 in. (5 $\mathrm{cm})$ ] as is planned for HESI.

Our method consists of expanding a laser beam and using the expanded beam as a surrogate for a largearea plane wave of hard $x$ rays, even though the RMC's are diffraction limited at visible wavelengths. A brief outline of how this is possible follows.

The principle that permits us to use visible laser light to analyze diffraction-limited grids was discovered in the 19th century, and then rediscovered by Cowley and Moodie in 1957.9 As discussed in detail more recently by Cowley, ${ }^{10}$ the essence of this principle is that a point source, when imaged with a grid having a sinusoidal transmission function, has multiple image planes (called Fourier images) where the image intensity exactly replicates the source intensity. For a periodic grid, the transmission function is a simple superposition of sinusoids, and in its image planes each harmonic of the Fourier image is a faithful reproduction of the corresponding harmonic of the source intensity distribution. One can reconstruct the total Fourier image by adding the harmonics, each multiplied by a phase factor of a very simple form (see Section 6.) By choosing the position of the rear grid to coincide with a convenient Fourier image plane of the forward grid, essentially we can eliminate the smearing effects of diffraction and correct for amplitude changes by using readily computed diffraction factors.

To implement this technique, the x-ray detectors are replaced with photomultiplier tubes (PMT's), and the modulated light intensity at the exit of the rear grid is measured as the grids rotate. The measured modulated light intensity is then Fourier analyzed to obtain its harmonic content. Comparison of the measured values of the harmonic content with theoretical values provides a quantitative measure of the imaging capabilities of the RMC's. This method was used to calibrate the coarser of the two RMC's, with 25-in. (64-cm) resolution, on the HEIDI payload prior to its balloon flight on 23 June 1993. Calibration of the other HEIDI RMC, with 11-in. (28-cm) resolution, 


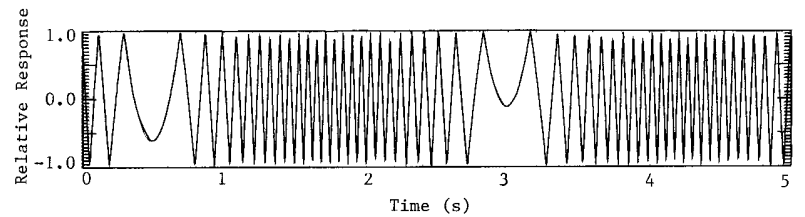

Fig. 2. Theoretical modulated signal that would be measured in one complete rotation about the RMC's axis of symmetry, observing an off-axis point source with an RMC that has a grid pitch of $1.25 \mathrm{~mm}$ and a grid separation of $5.2 \mathrm{~m}$. The maximum count rate in the plot was normalized to unity and offset to zero mean.

was not completed due to time constraints and other technical difficulties.

The advantages of our method are numerous. Angular resolution as fine as 2 arc sec is required to resolve the relevant physical features of impulsive solar flares with the HESI Fourier transform imager. The results we obtained with a laser and a inexpensive optical setup suggest that this approach readily can meet our angular resolution requirements with only minimal improvements in the optics. With the use of optical wavelengths, we remove any need for the use of a large-area plane wave of $x$ rays. Elimination of this requirement is not only a cost savings, but the elimination of the need to transport the instrument to a synchrotron beam or other facility is also a great savings in complexity and schedule. Our method of benchtop testing is preferable to observing celestial sources, such as stars or sunspots, because it also removes the necessity to take into account source variability and atmospheric blurring of the source, in addition to the obvious logistical difficulties of transporting the instrument to an acceptable observing location, suspending it, and pointing it in the required direction. Finally, this method also serves as an end-to-end test of the instrument's imaging optics, providing an assessment of the instrument's overall performance on a controlled, wellunderstood source distribution.

\section{X-Ray Imaging with Rotating Modulation Collimators}

Before discussing details of our calibration method, we present a brief overview of how x-ray images can be produced with RMC's. Figure 2 shows the theoretical modulated signal that would be measured in one complete rotation of an RMC about its axis of symmetry observing an off-axis point source. The RMC has a grid pitch of $1.25 \mathrm{~mm}$ and a grid separation of $5.2 \mathrm{~m}$. The maximum count rate in Fig. 2 was normalized to unity. The low-frequency portion of the modulated signal occurs when the slits or slats in the grid are nearly perpendicular to the projections of the line of sight to the x-ray point source onto the grid plane. In addition, the most rapid frequency variations occur when the slits or slats are nearly parallel to the same projection. This modulated signal contains all the information necessary to determine the location and intensity of the simulated point source, albeit with a point-spread function (PSF) of 25 in. (64 cm.).

There are a variety of methods that can be used for image reconstruction from this modulated signal. ${ }^{11-13}$ Even without reconstructing an image, for example, an estimate of the position of the point source can be inferred by inspection of Fig. 2. The number of cycles between the adjacent low-frequency portions of the signal is proportional to the angle between the rotation axis of the grids and the x-ray point source. The constant of proportionality is the pitch of the grids, expressed in arc minutes. There are 24 cycles between the low-frequency portions of the signal and, as stated above, the pitch of the grids is $1.25 \mathrm{~mm}$. A pitch of $1.25 \mathrm{~mm}$ with a grid separation of $5.2 \mathrm{~m}$ corresponds to an angular resolution of $25 \mathrm{in}$. $(64 \mathrm{~cm})$. Thus the x-ray point source is located at a radial distance of approximately $600 \mathrm{in} .(1524 \mathrm{~cm})$ [24 cycles $\times 25$ in. $(64 \mathrm{~cm})]$ or $10 \mathrm{ft}(3 \mathrm{~m})(24 \times 25 / 60)$ off the rotation axis of the RMC.

The time of the minimum in the low-frequency portion of the signal provides a measure of the azimuthal position of the x-ray source. The first minimum occurs at approximately $0.5 \mathrm{~s}$ in Fig. 2 . Because $5 \mathrm{~s}$ are required for one rotation of the RMC's, the x-ray source lies on a line that is rotated approximately $36^{\circ}$ $\left(360^{\circ} \times 0.5 \mathrm{~s} / 5 \mathrm{~s}\right)$ from the direction of the slits in the grids at time zero. The source is, therefore, $10 \mathrm{ft}$ (3 $\mathrm{m})$ off the rotation axis of the RMC and on a line approximately $36^{\circ}$ from the starting orientation of the grids. There is a $180^{\circ}$ ambiguity in the position of the source because the same pattern would be produced with the source on the opposite side of the spin axis.

The relative amplitudes of the local minima at approximately 0.5 and $3.0 \mathrm{~s}$ is a measure of the relative phase of the RMC, i.e., the projected distance between the center of rotation and the center of aperture in units of pitch. If the relative phase were zero, the values at these local minima (or maxima, depending on the source position) would be equal. The fact that the relative phase is nonzero indicates that the spin axis intersects the front and rear grids at different points in their respective grid coordinates.

Thus, for steady point sources, the position and intensity of the source and various instrument parameters can be inferred directly from the modulated signal. For multiple point sources or for sources with spatial extent, however, more complex instrumentation and processing are necessary. Ideally, an instrument should have as many RMC's as there are spatial scales in the sources to be mapped. Image reconstruction from the RMC responses is computer intensive, with expense roughly proportional to the square of the number of RMC's.

Conceptually, the simplest method of determining the PSF for a RMC is by back projecting the trajectories of an ensemble of photons passing through the grids from a hypothetical point x-ray source. ${ }^{14,15}$ This requires complete knowledge of the transmission functions of the individual grids and the colli- 

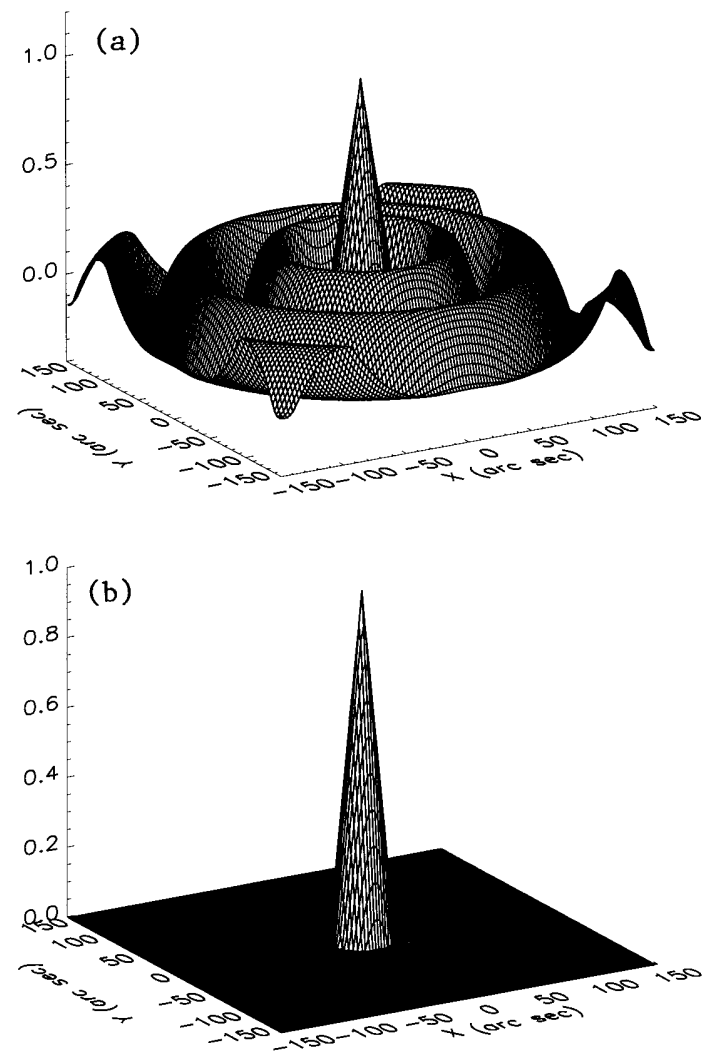

Fig. 3. (a) Computed map for a steady flux of $x$ rays from a hypothetical point source incident on an ideal 25 -in. (64-cm) collimator for one full rotation of the collimator about its axis. The collimator was assumed to be ideal and the (steady) source was taken to have the position used to make the modulation pattern of Fig. 2. We made a vertical shift in the map amplitudes to force the map to have zero mean. Except for the vertical shift, this figure and Fig. 2 are mutually compatible, and given the assumption of a steady source, either one could be used to derive the other. (b) Image of (a) postprocessed with the standard CLEAN algorithm used in radio astronomy to remove the sidelobes.

mator as a whole. In principle, at every instant during the rotation of the collimator, one can compute the transmission function of the collimator and project it on the sky. Each such projection corresponds to a probability map for that instant. To map the $\mathrm{x}$ rays from a source in the collimator's field of view, one superposes all the instantaneous probability maps computed for each time a photon is detected. The total probability map that results from this superposition is a dirty map with sidelobes that surround the true source. To first order, this is equivalent precisely to aperture synthesis in radio astronomy. For a point source, the probability map is the PSF of the instrument, or in radio astronomy parlance, the dirty beam.

We computed the probability maps for a uniform flux of $x$ rays incident upon the HEIDI 25 -in. $(64 \mathrm{~cm})$ collimator for one full rotation of the collimator about its axis. As above, the collimator was assumed to be ideal and the (steady) source was taken to have the position used to make the modulation pattern of Fig. 2. Figure 3(a) shows this probability map, with a

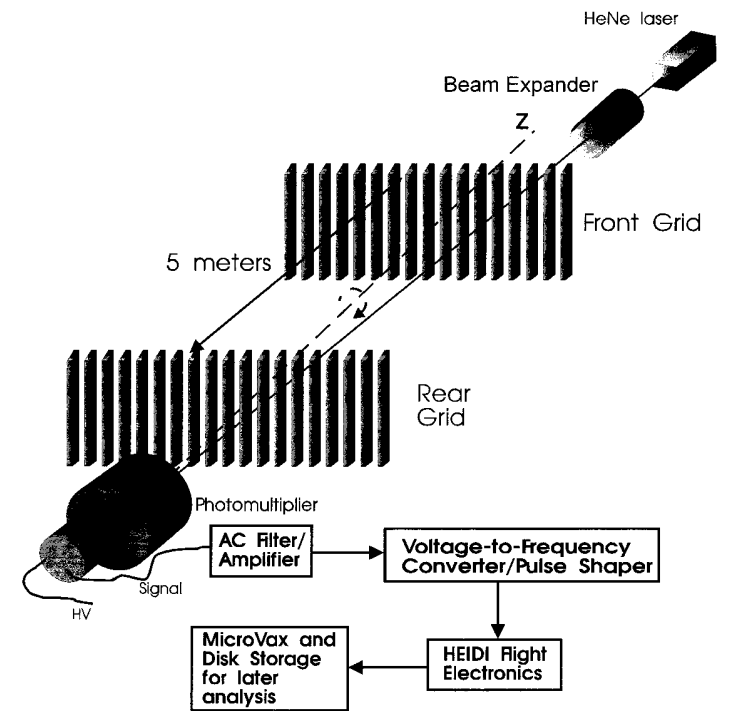

Fig. 4. Schematic of the RMC and electronics showing the experimental setup used in our calibration measurements. The beam from the He-Ne laser was directed through the eyepiece of a small Cassegrain telescope that expanded the beam. The plane wave that was generated intersected the front grid of the RMC at an angle of $12 \mathrm{ft}$. $(3.6 \mathrm{~m})$ relative to its rotation axis, labeled $\mathrm{Z}$. The light intensity that passes through both the front and the rear grids was measured with a PMT. The processing of the PMT signal is discussed in Section 3.

vertical shift in the amplitudes to force the map to have zero mean. Except for the vertical shift, Figs. 2 and 3(a) are mutually compatible, and given the assumption of a steady source, either one could be used to derive the other.

The central peak in the map of Fig. 3(a) is $25 \mathrm{ft}$ (64 $\mathrm{cm}$ ) wide, FWHM, and lies at the location of the presumed point source. The rings around the central peak would be reduced if probability maps from parallel collimators of different spatial resolution were added to this map, which is the motivation for the use of multiple collimators, as in the HESI. The tools of radio astronomy can be applied to such maps, and the sidelobes can be removed by the use of standard CLEAN $^{16}$ or maximum entropy method ${ }^{17,18}$ algorithms. Figure 3(b) shows the result of our applying CLEAN to Figure 3(a).

\section{Apparatus and Data Collection}

Figure 4 shows the experimental setup used in our calibration measurements. The beam from the $\mathrm{He}-\mathrm{Ne}$ laser, with a wavelength of $632.8 \mathrm{~nm}$, was directed through the eyepiece of a small Cassegrain telescope. The wave fronts from the beam-expanded laser simulated a point source at infinity. At optical wavelengths, the RMC's were diffraction limited, and the factor by which the modulated signal must be divided to account for diffraction effects is called the diffraction factor. (We discuss this factor in more detail in Section 6.) The telescope length was chosen so that the magnitude of the diffraction factor was not near zero at the laser wavelength used in our calibration. 
The plane wave that was generated had a diameter of approximately $70 \mathrm{~mm}$ and intersected the front grid of the RMC at an angle of $12 \mathrm{ft}(3.6 \mathrm{~m})$ relative to the rotation axis of the RMC (labeled the $\mathrm{Z}$ axis in Fig. 4). The diameter of both the front and rear grids used in our measurements is $130 \mathrm{~mm}$. The light intensity that passes through both the front and the rear grids was measured with a PMT. The PMT does not rotate with the grids. Because of the intensity of the laser light, the PMT could not be operated in a single photon-counting mode, but instead was operated in an analog output mode. Because the modulation pattern of the incident laser light by the grids was the object of interest (but not the average intensity level), the signal of the PMT was ac coupled to eliminate any dc background. The grids were then rotated about their axis of symmetry (the $\mathrm{Z}$ axis in Fig. 4), and the modulated light intensity observed with the PMT was converted into a voltage and passed through the ac coupling to a high-pass filter connected to the input of a voltage-to-frequency converter. The output of the voltage-to-frequency converter was a series of constant amplitude pulses whose frequency was proportional to the amplitude of the signal from the PMT. The pulses from the voltage-to-frequency converter were shaped electronically to simulate the signal that would be obtained with an x-ray detector and were then injected into the HEIDI flight data acquisition electronics. Here the individual pulses were time tagged with $100-\mu$ s resolution and stored. The data were later analyzed with the HEIDI MicroVax computer.

Although the experimental setup described above provided a modulated signal that can be observed even visually, gradients in the wave front produced by the relatively unsophisticated optics employed make different parts of the expanded laser beam appear to originate from a variety of different directions. To determine the degree of wave-front nonuniformity, additional measurements were made with an aluminum mask with a pattern of approximately 20 $\mathrm{mm}$-diameter holes drilled in it and was situated directly in front of the front grid. This mask was fixed relative to the incident wave front and did not rotate with the RMC's. It allowed light from only select portions of the wave front to illuminate the RMC. The centroid of the PSF reconstructed from data obtained with holes at different locations were compared to provide quantitative measures of the effects that were due to any nonuniformities in the incident plane wave. A perfect plane wave results in the centroid of the measured PSF appearing at the same location regardless of mask orientation. A plane wave with nonuniformities results in the centroid of the PSF appearing at a variety of locations depending on the mask orientation. Results from these measurements are presented in Section 7.

Stability of the entire experimental apparatus is also of extreme importance because any movement of the RMC with respect to the laser beam would result in smearing the centroid of the measured PSF. In our measurements the telescope pointing electronics

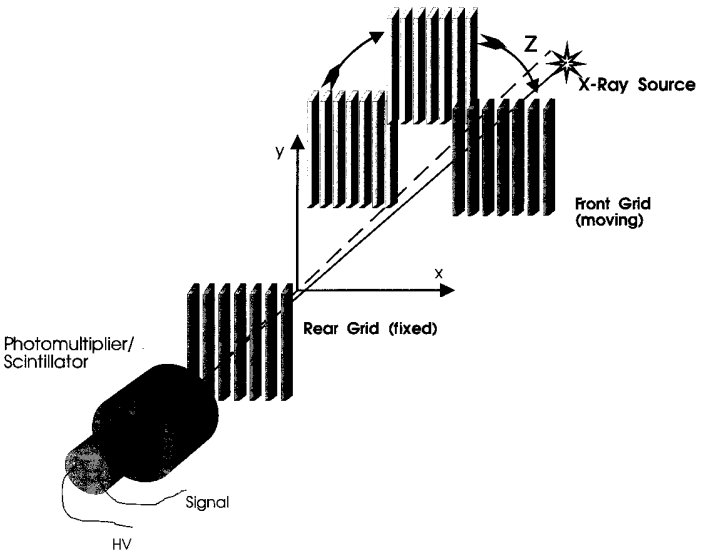

Fig. 5. For a point source located off the rotation axis, labeled Z, of the RMC's, the rear grid appears fixed and the front grid undergoes simple harmonic motion in both the $\mathrm{x}$ and the $\mathrm{y}$ directions centered around the $\mathrm{Z}$ axis. As discussed in the text, the motion in the $\mathrm{x}$ direction produces modulation that can be analyzed in one dimension.

and hardware were disabled so that the telescope housing the RMC's could be fixed rigidly in a horizontal position. Movement of personnel around the telescope during the calibration process was minimized so as to minimize distortions of the incident plane wave caused by air currents.

\section{Theoretical Harmonic Content}

One revealing way of visualizing the modulation produced by a RMC is as follows. (Our discussion here relates only to infinitely thin grids. The effect of finite thickness is discussed in Section 5.) The modulated signal through a RMC from an astronomical $\mathrm{x}$-ray point source that is not located on the rotation axis is the same as the signal that would be obtained if the observations were made with the source located directly over the center of the fixed rear grid and the front grid were moving on a circular path centered on the rotation axis. (If the source is not rotationally symmetric, it must rotate at the rate of the RMC, but for a point source, this is irrelevant.) The radius of this circular path is proportional to the radial position of the x-ray source relative to the rotation axis of the RMC. This arrangement is illustrated in Fig. 5. The front grid moves on a circular path around the axis of symmetry (the dotted line labeled Z). Note that the slits and slats of the front and rear grids remain parallel at all times as the front grid moves on its circular path. The motion of the front grid can be decomposed into two components, one parallel to the direction of the slits/slats (labeled y in Fig. 5) and one perpendicular to the slit/slat directions (labeled $\mathrm{x}$ in Fig. 5). The $\mathrm{x}$ and y axes in Fig. 5 are fixed relative to the rear grid. The front grid undergoes simple harmonic motion in both the $\mathrm{x}$ and the $\mathrm{y}$ directions. However, because the slits and slats of the front grid are aligned with the y direction, there is no modulation of the incoming $x$-ray flux due to motion in the $y$ direction. There is modulation of the x-ray flux due 
to motion in the $\mathrm{x}$ direction because the grids alternately occult and de-occult the source.

The relationship between $\mathrm{x}$ and rotation angle $\theta$ (the angle between the $\mathrm{x}$ axis shown in Fig. 5 and a vector pointing from the $\mathrm{z}$ axis to the center of the front grid that is located in the plane of the front grid) is given by

$$
\mathrm{x}=(R / p) * \sin \left(\theta-\theta_{0}\right),
$$

where $R$ is the radial distance of the source from the $\mathrm{z}$ axis shown in Fig. 5 (measured in units of pitch), $p$ is the pitch of the grids, and $\theta_{0}$ is the starting angle of the grids. The intensity (or equivalently the transmission through the two grids) versus $\theta$ results in a triangular waveform with a sinusoidally varying frequency as that shown in Fig. 2. (Note that the abscissa in Fig. 2 is not $\mathrm{x}$ but time, so the waveforms in Fig. 2 are not strictly triangular.) Plotting the intensity versus $x$ results in a triangular wave of constant frequency.

That the modulation gives an exact linear variation in transmission as a function of $\mathrm{x}$ can be understood as follows. The transmitted flux is proportional to the overlap of the slits in the upper and lower grids. This overlap is a series of rectangles that change linearly in width but stay fixed in length as the front grid moves in the direction perpendicular to the slats.

Regularization is the term used to describe the process of eliminating the sinusoidal frequency dependence of the modulated signal when the intensity versus $\mathrm{x}$ is plotted. ${ }^{11}$ The process of regularization corrects for the variable rate of modulation caused by rotation of the collimator. The measured modulated signal obtained from a perfect RMC can be regularized once the position of the source has been determined (see Section 7).

Fourier transforming the regularized waveform, i.e., a triangle wave of constant frequency, shows that for infinitely thin grids with equal slit and slat widths, the amplitudes of the odd harmonics are a factor of the square of the harmonic order times smaller than the fundamental. Thus the ratio of the fundamental to the third harmonic is 9 , the fundamental to the fifth harmonic is 25 , etc. The even harmonics for this case are identically zero.

The regularized triangular waveform is constructed from the sum of all the harmonics. A cosine waveform with the same frequency as the triangular waveform is the fundamental, or first-order approximation, to the triangle wave. The addition of further harmonics to the fundamental sharpens the peaks and valleys of the resulting sum. Imperfections in the grids, misalignment between the grids, systematic and random variations in pitch, etc. can manifest themselves as a degradation of the sharpness of the peaks and valleys in the modulated signal. This effect would be most observable in the amplitude of the third harmonic to the modulated signal. By a comparison of the measured ratio of the fundamental to the third harmonic with theoretical results, a
Table 1. Effect of Thickness on Harmonic Content

\begin{tabular}{lll}
\hline Harmonic & Ideal & Actual \\
\hline Fundamental & 1.0 & 1.0 \\
Second & 0 & $1 / 130$ \\
Third & $1 / 9$ & $1 / 9.6$ \\
Fourth & 0 & $1 / 135$ \\
Fifth & $1 / 25$ & $1 / 30.3$ \\
\hline
\end{tabular}

quantitative measure of the quality of the grids can be obtained.

\section{Effects that are Due to the Nonzero Thickness of the Grids}

The harmonic content described in Section 4 applies only to infinitely thin grids in which the slats are $100 \%$ opaque and that have a slit-width/slat-width ratio of unity. The actual grids used in the HEIDI instrument have a thickness of $10 \mathrm{~mm}$ that needs to be taken into account. The effect of the nonzero grid thickness is to modulate sinusoidally the effective transmission seen with an off-axis source as the RMC rotates about its axis of symmetry. When the grids are parallel to the line between the off-axis source and the projection of the spin axis on the sky, the optical transmission is a maximum. A quarter revolution later the transmission $[T(\phi)]$ is a minimum and is given by

$$
T(\phi)=[s-t * \tan (\phi)] / p,
$$

where $s$ is the slat width, $t$ is the thickness of the grid, $\phi$ is the off-axis angle of the source, and $p$ is the pitch. At intermediate grid orientations, the relative transmission is between $s / p$ and the value given by the above equation. The effect of the nonzero grid thickness manifests itself as a flattening of the bottoms of the triangle-shaped peaks that are due to the increased occultation of the source by the apparently thicker slats.

The harmonic content of the triangular waveform for 10-mm-thick grids is shown in Table 1 for the case $s=p / 2$ and $\phi=12 \mathrm{ft}(3.6 \mathrm{~m})$. The odd harmonics remain smaller than the fundamental by approximately the square of the harmonic order. Note that shadowing has introduced nonzero even harmonics. Although they are still much smaller than the contiguous odd harmonics, they can contribute significantly to the analysis. Higher harmonics than the fifth are sufficiently small that they may be lost in the noise, so we restrict much of our analysis to the second and third harmonics.

\section{Diffraction Effects}

Because the grids are diffraction limited at the wavelength at which the $\mathrm{He}-\mathrm{Ne}$ laser operates, the effects of diffraction on the measured modulated signal must be taken into account explicitly. These diffraction effects are negligible at x-ray energies above approximately $10 \mathrm{keV}$, but are not negligible in visible light. As mentioned in Section 1, we use a technique (de- 


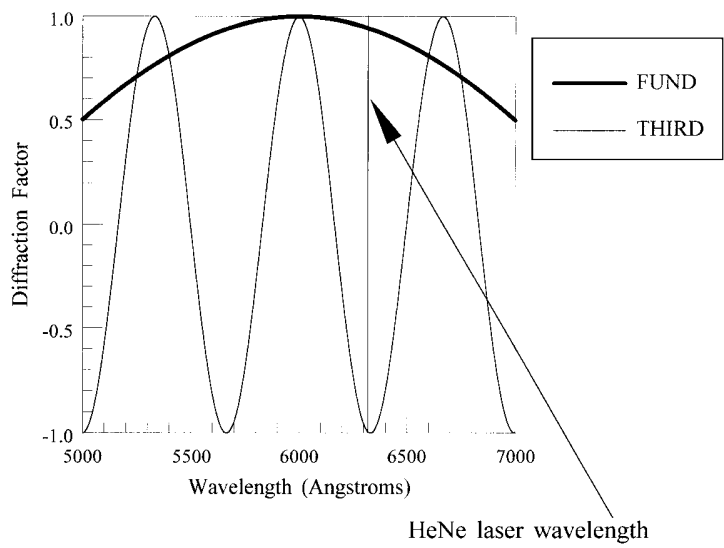

Fig. 6. Plot of the diffraction factor for the fundamental and the third harmonic versus wavelength for the RMC used in our calibration procedure (with a pitch of $1.25 \mathrm{~mm}$ and a grid separation of $5.2 \mathrm{~m}$ ). FUND, fundamental.

scribed by Cowley ${ }^{10}$ ) for mitigating the effects of diffraction, which is to place the rear grid in one of the Fourier image planes of the front grid. This would be sufficient to eliminate the effects of diffraction almost completely if the grids were ideal. Because they are not, further analysis is required.

For ideal bi-grid RMC's, Lindsey ${ }^{19}$ and Bar-Ziv ${ }^{20}$ have shown that the amplitude of the modulation time profile is multiplied simply by a diffraction factor $D$. For ideal grids, $D=1$ in the Fourier image planes; for imperfect grids, $D$ may never precisely be 1. The diffraction factor for ideal grids is

$$
D=\cos \left(\pi L h^{2} \lambda / p^{2}\right),
$$

where $L$ is the distance between the grids, $h$ is the harmonic order number, $\lambda$ is the wavelength of the incident plane wave, and $p$ is the pitch of the grids. Negative values of the diffraction factor correspond to phase shifts of $180^{\circ}$. Such a negative value results in the affected harmonic subtracting from, rather than adding to, the total modulated signal. A plot of the diffraction factor for the fundamental and the third harmonic versus the wavelength for the RMC used in our calibration procedure (with a pitch of 1.25 $\mathrm{mm}$ and a grid separation of $5.2 \mathrm{~m}$ ) are presented in Fig. 6. A derivation of the mathematical aspects of diffraction effects for irregular grids is presented in Section 7, where Eq. (3) is found as a special case [cf. Eq. (11) and the discussion that follows it].

Conventional diffraction limits are usually the shortest wavelength at which the amplitude of the measured signal is reduced by some significant amount. Figure 6 shows clearly, however, that at selected larger wavelengths (larger than the conventional diffraction limit), the effects of diffraction become negligible and the magnitude of the diffraction factor approaches unity. Physically this occurs when the separation of diffraction maxima formed by the front grid equals a multiple of the spacing of the rear grid. For a sufficiently narrow range of wavelengths, the effects of diffraction are constant and can be made negligible at optical wavelengths when appropriate values are chosen for the grid pitch and the distance between the grids. Such a range of wavelengths can be achieved with optical bandpass filters and white light or with laser light sources.

Figure 6 shows that the absolute values of the diffraction factors for the fundamental and the third harmonic are near maximum values at $632.8 \mathrm{~nm}$, the wavelength at which $\mathrm{He}-\mathrm{Ne}$ lasers operate. The diffraction factor for the fundamental is 0.945 , and the diffraction factor for the third harmonic is -0.989 . When the effects of diffraction are incorporated for perfect grids with a pitch of $1.25 \mathrm{~mm}$, the expected value of the fundamental-to-third-harmonic ratio is $-8.6: 1$. A measured ratio of $-8.6: 1$ with incident $\mathrm{He}-\mathrm{Ne}$ laser light would be consistent with a fundamental-to-third-harmonic ratio of 9:1 for $\times$ rays, for which diffraction effects are negligible.

Overall, the use of harmonics is a simple, straightforward way of interpreting the data. A more complete analysis of these data is beyond the point of diminishing returns. Future data obtained with a system described in Section 8 is expected to yield more precise and informative results.

\section{Application to the High-Energy Imaging Device Grids}

The first step in the analysis of the calibration data obtained for the HEIDI grids consists of building up a modulated signal with a sufficient signal-to-noise ratio and sufficient statistics to allow for a meaningful analysis. This is accomplished for the data sets, separately with and without masks, by the addition of all the simulated x-ray pulses counted by the HEIDI flight data-acquisition system, which is modulo $5 \mathrm{~s}$, the rotation period for the HEIDI grids. Effects produced by the electronic filter used to differentiate the signal with a time constant of 0.296 $\mathrm{s}$ are removed from the data, and the corrected rate is then normalized to a peak counting rate of unity with a mean of zero. The modulated photon rate $(\Phi)$, for runs without masks and for runs with masks, with each mask orientation, is fit to the following equation:

$$
\Phi=A * \cos \left[2 \pi(R / p) \sin \left(\theta-\theta_{0}\right)-\Delta\right],
$$

so as to determine the apparent position of the point source and the phase of the grids. Here, $p$ is the pitch of the grids, $R$ and $\theta$ are the radial and angular positions of the source, $\theta_{0}$ is the starting orientation of the grids relative to the source, $\Delta$ is the relative phase of the collimators, and $A$ is the amplitude of the modulated signal. (The collimator phase is the projected distance, in units of phase, of the spin axis from the line of zero phase for the RMC.) This equation is not intended to represent an exact analytic form of the modulated signal. The effects that are due to the finite thickness of the grids, grid imperfections, and diffraction effects are not taken into account in these fits. Rather, the intent of the fit is to find the centroid of the PSF, given by $R$ and $\theta$, and the instrument parameters $\theta_{0}$ and $\Delta$. 

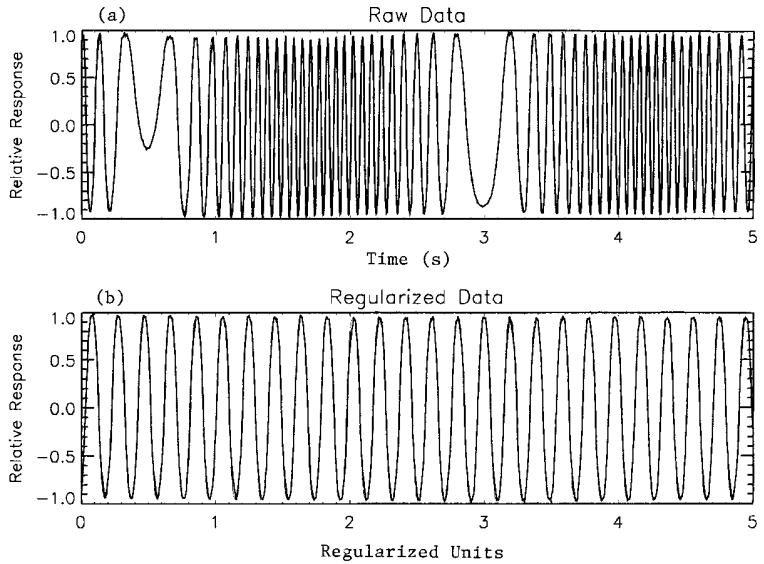

Fig. 7. Modulation profiles with derived empirical PSF and theoretical PSF: (a) raw data produced by the laser calibration system and (b) regularized data after correction for the variable rate of modulation caused by rotation of the collimator and after a best fit to the source position.

The best estimates for $R, \theta_{0}, \Delta$, and the mean grid pitch are used to regularize the modulated signal shown in Fig. 7(a), i.e., the modulated signal is plotted versus $2 \pi\left[(R / p) * \sin \left(\theta-\theta_{0}\right)+\Delta\right]$ to remove the sinusoidal frequency variation [Fig. $7(\mathrm{~b})]$. We use the regularized modulation profile to construct the instrumental PSF. When the phase of the grids is taken into account, the measured modulated signal also can be back projected through the grids to obtain a measured PSF (with the full extent of the laser beam). To do this, we replicate the profile (a function of $\mathrm{x}$ ) along the $\mathrm{y}$ axis to make an instantaneous two-dimensional PSF and then rotate the instantaneous two-dimensional PSF about a point $10 \mathrm{ft}(3 \mathrm{~m})$ from the center, successively summing the rotated versions at intervals of $2^{\circ}$ from $0^{\circ}$ to $360^{\circ}$ [Fig. 8(a)]. This produces a full rotation PSF. It is instructive to compare this with the theoretical PSF of Fig. 3. The rounded central peak in the empirical PSF is due primarily to a negative third harmonic, as discussed below.

The same procedure for runs with the masks in place provides a measured PSF for each orientation of the mask. The centroids of the measured PSF's for different locations of the mask apertures are then compared to seek inhomogeneities in the laser beam. The results of such a comparison are presented in Fig. 9, which shows that the apparent relative position of the centroid of the measured PSF varies by approximately $2 \mathrm{in}$. $(5 \mathrm{~cm})$, or approximately $10 \%$ of the grid pitch of $25 \mathrm{in}$. $(64 \mathrm{~cm})$, depending on the location of the hole in the mask. The position shift was correlated angularly with the position of the mask hole. This is probably an indication of nonflat wave fronts in the expanded laser beam at its incidence upon the front grid. Because the departures from planarity are found to be small $[\ll 25$ in. (64 $\mathrm{cm})$ ], all subsequent analysis uses data obtained with no masks so as to keep the signal-to-noise ratio as high as possible.
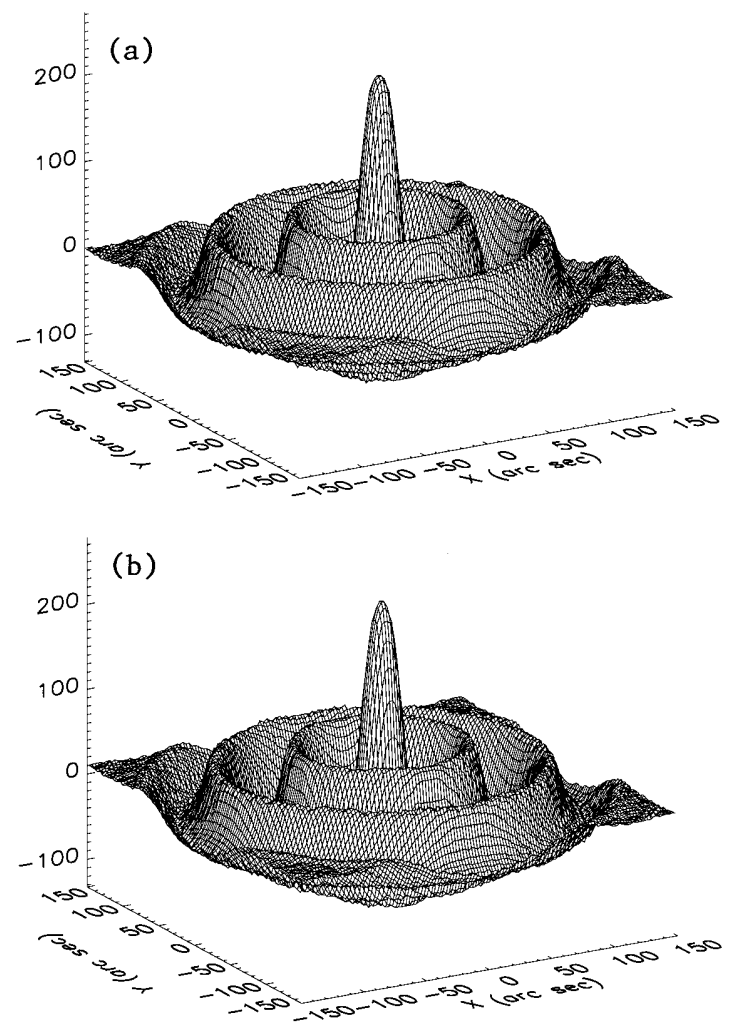

Fig. 8. (a) Empirical PSF deduced from Fig. 7(b). Compare this with the ideal PSF shown in Fig. 3(a). The rounded shape of the central peak was produced by the reversed sign of the third harmonic, one of the effects of diffraction. (b) Theoretical PSF with the fundamental, second, and third harmonics determined from analytic fits to the curve in Fig. 7(a) and including the diffraction factors. Comparison of this PSF with the empirical PSF in (a) shows only slight differences, mainly attributable to grid irregularities and the neglect of higher harmonics.

Once the (full-beam) modulated signal is regularized, a fast Fourier transform routine determines its harmonic content, which consists of a discrete line

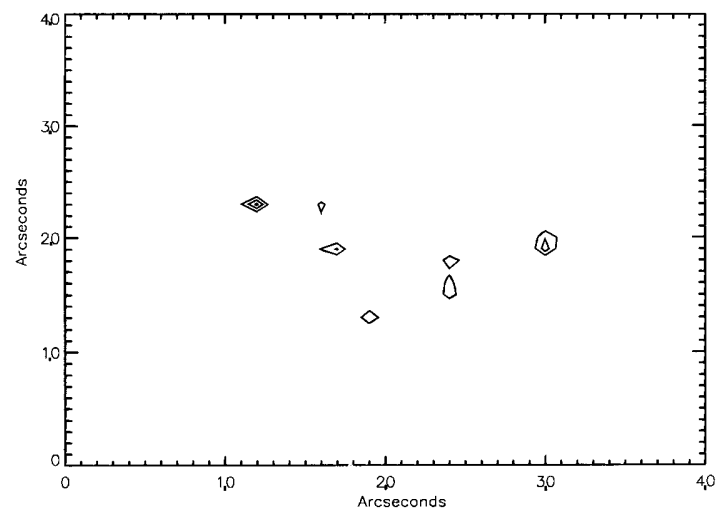

Fig. 9. Apparent positions of the point source for different locations of the mask apertures. The apparent relative position of the point source varies by approximately $2 \mathrm{in} .(5 \mathrm{~cm})$ or approximately $10 \%$ of the grid pitch of $25 \mathrm{in}$. $(64 \mathrm{~cm})$, depending on the location of the hole in the mask. The position shift was correlated angularly with the position of the mask hole. 


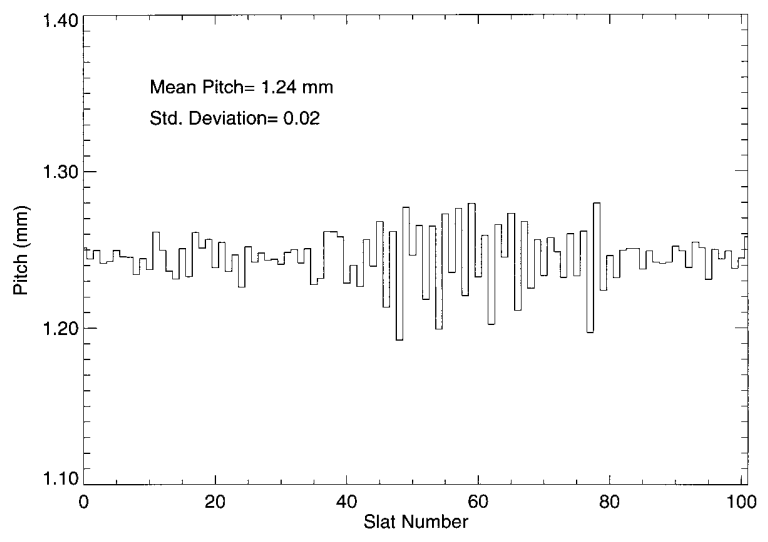

Fig. 10. Histogram of the measured variation in pitch across one of the HEIDI grids. The distribution in grid pitch is of the order of $10 \%$ of the mean grid pitch of $1.25 \mathrm{~mm}$.

spectrum. The first and second halves of the collimator rotation provide two distinct and complete measurements of the modulation spectrum. For the third harmonic, the ratios to the fundamental $\Re_{3: 1}$ for the two halves are found to be -0.065 and -0.069 , implying that $\Re_{3: 1}=-0.067 \pm 0.002$. The second harmonic is less well determined, with a range determined to be from 0.020 to 0.030 , and so $\mathfrak{R}_{2: 1}=$ $0.025 \pm 0.010$. For ideal grids at near-normal incidence, the magnitude of the third harmonic ratio $\left|\Re_{3: 1}\right|$ is smaller than expected and $\Re_{2: 1}$ is larger than expected. We discuss the reasons for these discrepancies below.

With the above empirical harmonic ratios, it is now possible to construct a theoretical PSF [Fig. 8(b)] for comparison with the empirical PSF of Fig. 8(a). Inspection of Figs. 8(a) and 8(b) shows negligible differences between them, validating the three-harmonic approximation and indicating the good quality of the RMC.

An independent visible light characterization of each of the grids that comprise the RMC used in our calibration procedure was performed prior to the construction of the RMC. Figure 10 shows a histogram of the measured variation in pitch across one of these grids. The distribution in grid pitch has a FWHM of the order of $10 \%$ of the mean grid pitch of $1.25 \mathrm{~mm}$.

The theoretical value of the fundamental-to-thirdharmonic ratio given above incorporates only the mean value of the pitch. Because the diffraction factor varies as the $\cos \left(C / p^{2}\right)$, the variations in pitch can have a dramatic effect on the value of the diffraction factor. The variation in pitch therefore must be taken into account when the diffraction factor is determined. A derivation of expressions for the effects of diffraction on a plane wave of monochromatic light on a collimator with irregular grids follows.

\section{A. Analysis of Modulation by Irregular Grids}

For a periodic system of apertures, assumed symmetric about the origin, one can write the aperture transmission function in the form of the following Fourier series:

$$
q(x)=\sum_{h} F_{h} \cos (2 \pi h x / p), \quad h=1,3,5, \ldots,
$$

where $p$ is the period of the aperture (pitch), and the harmonics $h$ run from 0 to $\infty$. In this particular case, $F_{h}=\sin (h \pi / 2) / h(h \neq 0)$ and $F_{0}=1 / 2$. This form of the transmission function is well suited for computing the effects of diffraction (see Ref. 20). In the real world, however, grids depart from the ideal in both symmetry and periodicity. For irregular grids without any symmetry, the above equation must be generalized to

$$
\begin{aligned}
q(x) & =\sum_{n} F_{n} \exp (2 i \pi n x / d), \\
n & =-M,-M+1, \ldots, M,
\end{aligned}
$$

where the fundamental period is now $d$, the width of the grid rather than the pitch, and $M \gg$ the number of slats. This form is used for the forward grid, and for the rear grid a similar form is used $[\hat{q}(x)$ replaces $q(x)$ and $\hat{F}_{n}$ replaces $F_{n}$, and the $x$ coordinate is offset by an arbitrary amount $\delta]$. Note that the functions $q(x)$ and $\hat{q}(x)$ are all zeroes or ones, hence they are equal to their own square $\left(q^{2}=q\right)$, which implies that the autocorrelation of their Fourier coefficients is identically the same sequence as that of the Fourier coefficients. This important property is used below as a check on the zero-wavelength limit of our final expression.

The number of coefficients $F_{n}$ required to reproduce the Goddard Grid Characterization Facility (GCF) measurements $(10-\mu \mathrm{m}$ accuracy) is approximately $100 \times N(N=$ number of slats $)$, but for our purposes here, $\sim 20 \times N$ (4096 coefficients) is sufficient.

Then, as shown by the standard method of Fourier transforming the aperture transmission function (e.g., Cowley ${ }^{10}$ ), one can compute the $n$th harmonic of the electric vector of a wave after passage through the grid:

$$
\psi_{n}(x)=\exp (-i k L) F_{n} \exp \left(i \pi L \lambda n^{2} / d^{2}\right) \exp (2 i \pi n x / d),
$$

where $L$ is the distance of the image from the aperture plane, $\lambda$ is the wavelength, and $k=2 \pi / \lambda$. This is essentially Eq. (31) in Cowley, ${ }^{10}$ which is valid in both the Fresnel and the Fraunhofer domains. Note that at locations where $L$ is an integer multiple of $2 \lambda n^{2} / d^{2}, \psi_{n}(x)$ assumes the same values as the zerowavelength limit. These locations are the so-called Fourier image planes.

The intensity distribution of the image is the absolute squared sum of the phasers $\psi_{n}(x)$, which can be written as the Fourier series:

$$
\begin{aligned}
I(x)= & F_{0}^{2}+\sum_{n \neq 0} \cos \left(\pi L \lambda n^{2} / d^{2}\right) F_{n} \exp (2 i \pi n x / d) \\
& +\sum_{n \neq 0} \exp (i \pi n x / d) \sum_{m \neq 0} G_{m} G_{m+n}{ }^{*},
\end{aligned}
$$

where $*$ indicates the complex conjugate, and $G_{m}$ is a phase-shifted Fourier coefficient $F_{m} \exp \left(i \pi L \lambda m^{2} /\right.$ $d^{2}$ ). 
If the second grid of a collimator is placed in the image plane (at distance $L$ from the first grid), then modulation is observed when one displaces the second grid by an amount $\delta$ with respect to the first and measures the flux of photons passing through as a function of $\delta$.

The flux $\Phi$ through this second set of apertures is given when one integrates the intensity distribution over the extent of the grid, $-d / 2<x<d / 2$ :

$$
\Phi(\delta)=\int_{-d / 2}^{d / 2} d x \hat{q}(x) I(x) .
$$

This integral is evaluated readily with the fundamental orthogonality relation for Fourier series. Applying this to Eq. (9), one obtains the flux as a function of displacement $\delta$ :

$$
\begin{aligned}
\Phi(\delta)= & d \sum_{m} \sum_{l} \hat{F}_{l-m} F_{m} F_{l}^{*} \exp \left(i \pi L \lambda m^{2}-l^{2} / d^{2}\right) \\
& \times \exp (-2 i \pi m-l \delta / d) .
\end{aligned}
$$

For the case of an ideal periodic grid, except for the dc term $(n=0)$, the only harmonics that would appear in the aperture transmission functions $q(x)$ and $\hat{q}(x)$ are odd $(n=1,3,5, \ldots)$. But an examination of Eq. (10) shows that the only cases when all of the coefficients $\hat{F}_{l-m}, F_{m}$, and $F_{l}^{*}$ are nonzero is when one of $l$ or $m$ vanishes. Physically this is equivalent to one stating that for modulation to occur, the image of the first grid must be phase locked with the second grid.

When Eq. (10) is applied to an ideal grid of pitch $p$ with unit slit/slat ratio for harmonics $h=1,3,5, \ldots$, the flux reduces to

$\Phi_{h}^{\text {ideal }}(\delta)=4 d\left|\hat{F}_{h} F_{h}{ }^{*}\right| F_{0} \cos (2 \pi h \delta / p) \cos \left(\pi L \lambda h^{2} / p^{2}\right)$.

The modulation is a sinusoidal function with period $\delta=$ $p / h$ and amplitude proportional to $\cos \left(\pi L \lambda h^{2} / p^{2}\right)$. Thus the special case of regular grids reduces to the result found by Lindsey, ${ }^{19}$ alluded to above in Section 6 .

To apply Eq. (10) we must determine the coefficients $F_{n}$ and $\hat{F}_{n}$ for the grids. This is done when we employ the measurements of the slat positions made at the Goddard GCF. For each grid, positions of the slat edges were determined on ten parallel scans perpendicular to the slats. For all practical purposes these scans are identical. We designate these edge positions by $a_{k}$ and $b_{k}$ for the forward grid and $\hat{a}_{k}$ and $b_{k}$ for the rear grid.

The Fourier coefficients are then determined from the following equations:

$$
\begin{aligned}
F_{n}= & (1 / 2 \pi i) \sum_{k=1}^{N}\left[\exp \left(-2 i \pi n a_{k} / d\right)\right. \\
& \left.-\exp \left(-2 i \pi n b_{k} / d\right)\right] / n, n \neq 0 \\
F_{0}= & (1 / d) \int q(x) d x=\text { effective transparency, }
\end{aligned}
$$

with corresponding expressions for $\hat{F}_{n}$. Note that $F_{-n}=F_{n}{ }^{*}$, as expected for real $q(x)$. Also, even for irregular grids, $F_{n}$ is its own autocorrelation.

Because we are interested particularly in the modulation at the fundamental and the third harmonic of the mean grid pitch, we need to extract the part of the modulation in Eq. (10) that varies sinusoidally with a given harmonic of the rear grid offset $\delta$. Toward this end, we rewrite Eq. (10) as

$$
\Phi(\delta)=\sum_{n} \Phi_{n} \exp (-2 i \pi n \delta / d) .
$$

For a mean pitch $\bar{p}$, the fundamental occurs at $n=$ $d / \bar{p}$ and the second and third harmonics at $n=2 d / \bar{p}$, $3 d / \bar{p},\left(N_{1}, N_{2}\right.$, and $N_{3}$, respectively). A comparison of Eqs. (9) and (6) gives the expression for $\Phi_{n}$ :

$$
\Phi_{n}=d \hat{F}_{n} * \sum_{m} G_{m} G_{m+n} *
$$

where

$$
G_{m}=F_{m} \exp \left(i \pi L \lambda m^{2} / d^{2}\right) .
$$

Thus $\Phi_{n}$ is proportional to the autocorrelation of $G_{m}$, derived from phase shifts of the aperture coefficients of the forward grid. In the short wavelength limit ( $\lambda$ $=0), G_{m}=F_{m}$, so

$$
\Phi_{n}=d \hat{F}_{n} * \sum_{m} F_{m} F_{m+n} *=d \hat{F}_{n} * F_{n}, \lambda \ll d^{2} / L,
$$

where we use the fact mentioned above that the autocorrelation of $F_{m}$ equals $F_{m}$ itself. This reproduces a well-known result from geometrical optics: The Fourier transform of the image from two successive apertures is the product of the transforms of the apertures.

It is possible to manipulate Eq. (14) so that it is more clearly a generalization of Eq. (11) for ideal grids:

$$
\Phi_{n}=d \hat{F}_{n}^{*} \sum_{m=-\infty}^{\infty} F_{m} F_{m+n} * \cos \left(n+2 m n \pi L \lambda / d^{2}\right) .
$$

For the odd harmonics, this reduces simply to Eq. (11), but the algebra of the even harmonics is more subtle, and we found that it is more instructive to use Eq. (14) or (16) in numerical form.

\section{B. Comparison of Laser Calibration with the Grid} Characterization Facility Measurements

The coefficients $\Phi_{n}$, where $n=N_{1}, N_{2}$, and $N_{3}$, can now be used to obtain the amplitude and phase of the second and third harmonics relative to the fundamental. For any reasonably good grid, the dominating coefficients $F_{n}$ are the dc, fundamental, and third harmonic terms $n=0, n=N_{1}$, and $n=N_{3}$. (If the slit and slat widths differ, the significance of the second harmonic is additionally enhanced.)

Figure 11 shows the value of the diffraction factor for the fundamental and third harmonics for a range of pitches near $1.250 \mathrm{~mm}$. This figure clearly demonstrates the strong dependence of the diffraction fac- 


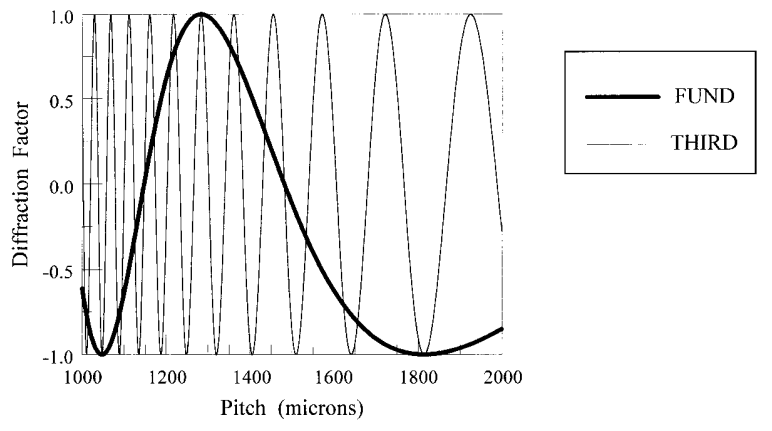

Fig. 11. Diffraction factor for the fundamental and third harmonics for a range of pitches near $1.250 \mathrm{~mm}$. This plot demonstrates the strong dependence of the diffraction factor on grid pitch.

tor on grid pitch. The equations for the dependence of the diffraction factor as derived above were used to compute the ratio of the harmonics to the fundamental $\left(\mathfrak{R}_{2: 1}\right.$ and $\left.\Re_{3: 1}\right)$, including grid irregularities. Equation (10) was used to obtain $\Phi_{n}$ for values near $N_{1}, N_{2}$, and $N_{3}$, then Gaussians were fit to the peaks and integration under the Gaussians was computed. The ratios of the products of these integrals for the fundamental and harmonics then gave the desired quantities $\Re_{2: 1}$ and $\Re_{3: 1}$.

If it is assumed that the grids are illuminated fully by the laser beam. Then, taking into account diffraction from grids with a variation in pitch, the empirical $\Re_{3: 1}$ is $-1 / 10.5$ and $\Re_{2: 1}$ is $1 / 40$. However, the expanded beam was approximately $88 \mathrm{~mm}$ in diameter, so approximately 70 slats of the front grid were projected onto the rear grid. However, this does not include the two-dimensional nature of the grid: In fact, because the mean chord of a circle is $\pi / 4$ times its diameter, the average number of slats illuminated by the approximately circular beam is approximately $\pi / 4 \times 70=55$. The area covered by the expanded laser beam is also reduced by the shadow of the secondary, and this further reduces the effective number of illuminated slats by several percent. For the case of illumination of the inner 51 slats, $\left|\Re_{3: 1}\right|$ decreases to $1 / 11$ and $\Re_{2: 1}$ increases to $1 / 30$. These changes result from the fact that the departures of the pitch from the nominal are larger in the middle of the grid.

There are a number of instrumental parameters that raise or lower the harmonic ratios. We already discussed the effect of pitch variations. Another important parameter is the effective slit/slat ratio. The slit/slat ratio, as measured at the GCF, is close to unity for the two coarse HEIDI grids. However, the off-set angle of the source from the spin axis creates slit shadowing that decreases the projected slit size in the detector plane. As discussed in Section 5, for ideal thick grids, this increases the even harmonics. For the source off-set angle of $12 \mathrm{ft}(3.6 \mathrm{~m})$, the slits are reduced effectively by $0.035 \mathrm{~mm}$. Other effects, such as the nonperpendicularity of the grids relative to the spin axis and venetian blinding in the grids, also cause effective reduction of the slit width and change the harmonic ratios. Therefore we took

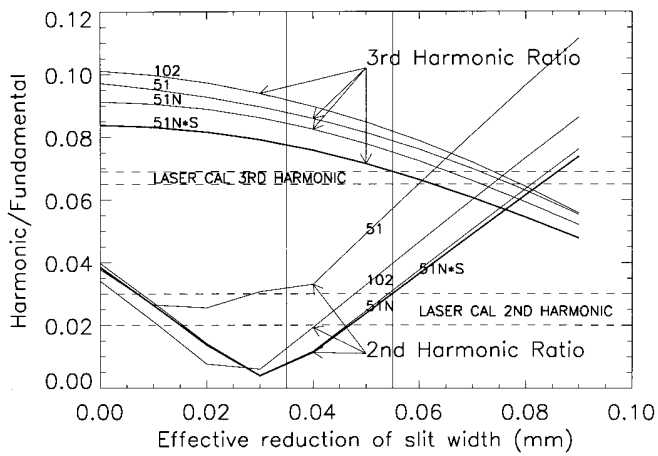

Fig. 12. Computed harmonics as a function of hypothetical reduction in slit width by shadowing, including the effects of diffraction and pitch irregularities. The amplitudes of the harmonics inferred from the regularization of the laser modulation signal are shown as horizontal bands bordered by dashed lines. The curves labeled 102 and 51 are for illumination of the full grid (102 slats) and for the inner half, respectively. The curves labeled $51 \mathrm{~N}$ are for 51 illuminated slats with the addition of $0.020-\mathrm{mm}$ noise to the aperture transmission function. The dark curves labeled $51 \mathrm{~N} * \mathrm{~S}$ include the effect of bandwidth smearing, possibly caused by twists and grid curvature, totaling $25 \%$ of the pitch. The left vertical line shows the maximum reduction of slit width caused by the 12 $\mathrm{ft}(3.6 \mathrm{~m})$ offset of the source, and the right vertical line shows the inferred effective reduction of slit width, where the computed harmonics (heavy curves) agree with the observations (dashed lines).

the effective slit reduction as a free parameter $(\Delta s)$, and computed the second and third harmonic ratios to the fundamental as a function of $\Delta s$. These quantities are shown in Fig. 12.

As the curves in Fig. 12 show, the ratio of the second harmonic to the fundamental $\mathfrak{R}_{2: 1}$ first falls and then increases as shadowing increases. The initial decrease results from the fact that one of the grids has a slat width slightly greater than its slit width. For ideal symmetric grids, the second harmonic would increase steadily from zero. Figure 12 shows that the third harmonic ratio $\left|\Re_{3: 1}\right|$ decreases monotonically as shadowing increases. For zero shadowing $(\Delta s=0)$, the third harmonic for an ideal grid is 0.11 , as shown by the top curve (labeled 102) for the case of 102 illuminated slats. For the case of 51 illuminated slats, the third harmonic as a function of $\Delta s$ decreases in parallel fashion. In general, as shown in Fig. 12, when the number of slats are reduced it causes the third harmonic to decrease and the second harmonic to increase. (Compare curves labeled 51 with those labeled 102.)

We found empirically that the presence of noise in the edge values increases the magnitudes of both $\Re_{3: 1}$ and $\Re_{2: 1}$. The curves labeled $51 \mathrm{~N}$ show the effect of our adding $0.020 \mathrm{~mm}$ pseudorandom noise to the aperture transmission functions. This value is approximately twice the noise expected from the variations in the GCF measurements at that time. Presumably, noise in the laser wave fronts and in the planarity of the beam would also cause such changes in $\Re_{3: 1}$ and $\Re_{2: 1}$. Because we know that the beam is nonplanar (see Fig. 9), we use this pseudorandom noise as a rough model of the beam aplanarity. 
Other important effects on the harmonic ratios involve the relative twists of the fore and aft grids and curvature of the grid slats. (Indeed, slat curvature was measured at the GCF and found to be a few percent of the nominal pitch.) Minor collimator twists and curvature have the effect of decreasing the modulation by a mechanism similar to bandwidth smearing (as it is called in radio astronomy). One may approximate this phenomenon by considering elemental differential sections of the collimator where the thin dimension lies in the direction perpendicular to the slats and to the spin axis. Each such differential element contributes to the overall modulation pattern, and if there is a small collimator twist or slat curvature, the pattern of each contribution will be phase shifted with respect to the other elemental modulation patterns. Thus the total of near-ideal sinusoidal patterns from each element will be smeared out. We model this by assuming that all elements contribute equally, and that the total sinusoidal modulation is a uniform contribution from phase shifts between 0 and $\alpha$. It is shown easily that each harmonic $N$ is reduced in amplitude by a smearing factor $S_{b}=\sin (N \alpha) / \alpha$. Figure 12 shows the harmonic ratios reduced by this factor (dark curves labeled $51 \mathrm{~N}^{*} \mathrm{~S}$ ) for the case $\alpha=0.25$. This $\alpha$ corresponds to maximum twist or curvature displacements of $25 \%$ of the pitch. Larger values than this were not computed because the approximation used here breaks down, and such large values seem unlikely.

Figure 12 shows the amplitudes of the harmonics inferred from the regularization of the laser modulation signal (horizontal bands bordered by dashed lines), as discussed in Section 6 . The predicted third harmonic ratio intersects the laser measurement lines at values of the abscissa for $\Delta s \approx 0.055 \mathrm{~mm}$, which is larger than the effective shadowing of 0.035 $\mathrm{mm}$ expected for a source offset by $12 \mathrm{ft}(3.6 \mathrm{~m})$. The left vertical line shows the maximum reduction of slit width caused by the $12-\mathrm{ft}(3.6-\mathrm{m})$ offset of the source, and the right vertical line shows the inferred effective reduction of the slit width where the computed harmonics (dark curves) agree with the observations (dashed bands). A possible explanation for the separation of the two vertical lines could be a combination of a slight lack of parallelism of the planes of the front and rear grids and/or more venetian blinding in the grids than anticipated.

The parameters selected to create this agreement (illumination fraction, noise, shadowing, and smearing) are not determined uniquely by our method, but we demonstrated the utility of laser calibration for the estimation of such optical parameters of the telescope. An improved system that is capable of measuring higher harmonics with higher accuracy would be able not only to provide an end-to-end test of the modulation, as we have done, but also to determine more precisely many of the relevant instrumental parameters.

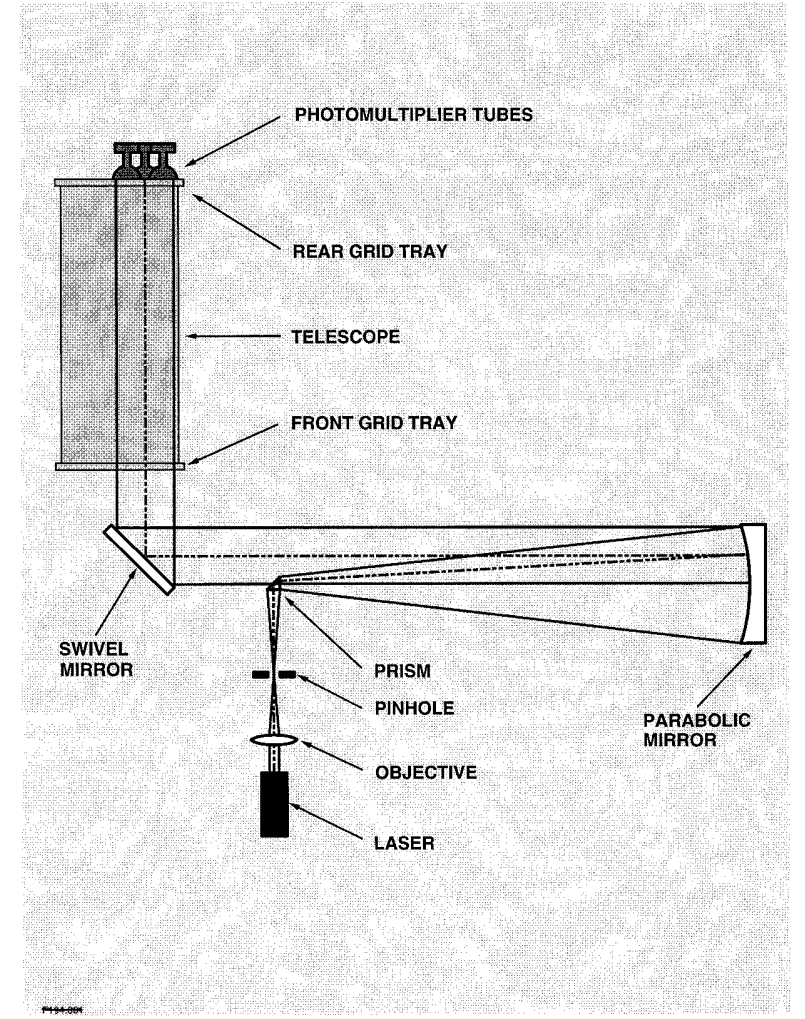

Fig. 13. Schematic of proposed improvements to laser calibration setup. The collimator we propose to use consists of an objective lens, a pinhole for spatial filtering, and a large parabolic mirror instead of an inverted Cassegrain telescope. The parallel, largearea beam reflected from the parabolic mirror will then be reflected onto the front grid of the RMC with a large-area planar mirror that is computer controlled. CAL, calibration.

\section{Modifications and Improvements}

Based on our experience with the first laser calibration of a HEIDI RMC, we proposed an improved experimental setup for a laser calibration system. The collimator we propose to use consists of an objective lens, a pinhole for spatial filtering, and a large parabolic mirror instead of an inverted Cassegrain telescope. The narrow laser beam would be focused by the objective lens onto a point in the plane of the pinhole. The pinhole would act as a spatial filter for high-frequency noise that is due to atmospheric scatterers, lens imperfections, etc. that will remain unfocused in an annular region about the center of the pinhole. After the pinhole, a prism will be used to direct the diverging beam toward a parabolic mirror. The prism will be situated such that the diverging beam will appear to have originated at the focal point of the mirror. The parallel, large-area beam reflected from the parabolic mirror will then be reflected onto the front grid of the RMC with a largearea planar mirror. A schematic of our proposed experimental setup is shown in Fig. 13.

The parallel beam produced in this manner, which will be of much higher quality than that produced by an inverted Cassegrain telescope, will allow our method of laser calibration to be applied to the finer 
grids currently being developed for the HESI instrument. Because of the higher beam quality, it also would permit a more complete analyses of future measurements than were possible with the results of our present system.

\section{Summary and Conclusions}

We developed a method to calibrate the imaging capabilities of rotating modulation collimators (RMC's) used as the imaging optics in astronomical Fourier transform x-ray imagers. Our method, which exploits the properties of Fourier image planes, can be performed in the laboratory with visible light (even though the grids that comprise the RMC are diffraction limited at optical frequencies) and does not require a large-area x-ray beam. We applied our method to one of the RMC's used in the high-energy imaging device (HEIDI) prior to its first balloon flight in June 1993. Our results for this RMC, with 25-in. $(64-\mathrm{cm})$ resolution, yield second and third harmonic ratios to the fundamental of 0.025 and 0.067 , respectively. After accounting for diffraction appropriately, which included incorporating the known irregularities in the grids, we compared the predicted ratios of the harmonics of the second and third modulation relative to the fundamental with the ratios derived from regularization of the laser modulation signal. The comparison indicates agreement between predictions and measurement if there are very slight angular offsets from the nominal in the two grids and their slats. The estimated values for these offsets are compatible with good overall imaging quality of the HEIDI collimator.

C. C. Gaither acknowledges the support of the National Academy of Sciences/National Research Council Research Associateship Program. C. N. Hartman acknowledges the support of the NASA Goddard Space Flight Center (GSFC) Code 730 (especially D. Krueger, D. Dalton, and D. Andrucyk). We are indebted to the GSFC Space Technology Division, Code 710 (Stephen Graham and Jeffrey Travis) for the test electronics. We are also indebted to the GSFC Optics Branch, in particular to John Ostankowski for advice and encouragement with this effort and to Paul Hannon for the schematic design of the advanced laser calibration facility. We thank Julie Ann Watko for assistance with the facility layout and sizing the components. This research was supported in part at the GSFC by NASA Research and Technology Operating Plan 370-04-07 and by the National Science Foundation/Research Experiences for Undergraduates site grant ATM 9400746. At the Catholic University of America, this research was supported in part by NSG 5066. At Caltech this research was supported in part by NAS 5-30792. At the University of Maryland this research was partially supported by NASA grant NAG 5-2001.

\section{References}

1. H. S. Hudson, K. T. Strong, B. R. Dennis, D. Zous, M. Inda, T. Kosugi, and T. Sakao, "Impulsive behavior in solar soft x-radiation," Astrophys. J. 422, L25-L27 (1994).
2. S. Masuda, T. Kosugi, H. Hara, S. Tsuneta, and Y. Ogawara, "Loop-top impulsive hard x-ray source of a solar flare as evidence of magnetic reconnection," Nature (London) 371, 495497 (1994).

3. T. Sakao, "Characteristics of solar flare hard x-ray sources as revealed with the hard x-ray telescope aboard the Yohkoh satellite," Ph.D. dissertation (University of Tokyo, Tokyo, Japan 1994).

4. B. R. Dennis, R. P. Lin, R. C. Canfield, C. J. Crannell, A. G. Emslie, G. D. Holman, H. S. Hudson, G. J. Hurford, J. C. Ling, N. W. Madden, and R. Ramaty, "The High Energy Solar Spectroscopic Imager-HESSI," in Missions to the Sun, D. M. Rust, ed., Proc. SPIE 2804 (1996).

5. C. J. Crannell, B. R. Dennis, L. E. Orwig, E. J. Schmahl, F. L. Lang, R. Starr, J. P. Norris, M. E. Greene, G. J. Hurford, W. N. Johnson, and K. S. Wood, "A balloon-borne payload for imaging hard x-rays and gamma rays from solar flares," in Proceedings of the AIAA International Balloon Technology Conference (American Institute of Aeronautics and Astronautics, New York, 1991).

6. M. Pelling, P. T. Feffer, K. Hurley, S. R. Kane, R. P. Lin, S. McBride, J. H. Primbsch, D. M. Smith, K. Youseffi, G. Zimmer, F. Cotin, J. M. Lavigne, G. Rouaix, S. Slassi, G. Vedrenne, R. Pehl, C. Cork, P. Luke, N. Madden, and D. Malone, "A high resolution gamma-ray and hard X-ray spectrometer (HIREGS) for long duration balloon flights," in EUV, X-Ray, and GammaRay Instrumentation for Astronomy III, O. H. Siegmund, ed. Proc. SPIE 1743, 408-421 (1992).

7. K. Makishima, "Instrumental performance of the SXT collimator," in Hinotori Symposium on Solar Flares (Institute of Space and Astronautical Science, Tokyo, 1982), pp. 120-129.

8. K. Makishima, Y. Ogawara, S. Miyamoto, N. Muranaka, and M. Oda, "Alignment of modulation collimator grids using optical diffraction patterns," ISAS Research Note 29 (Institute of Space and Astronautical Science, Megura, Japan, 1977).

9. J. M. Cowley and A. F. Moodie, "Fourier images. I. The point source," Proc. Phys. Soc. London Sect. B 70, 486-496 (1957).

10. J. M. Cowley, Diffraction Physics (Elsevier, New York, 1981).

11. H. W. Schnopper, R. I. Thompson, and S. Watt, "Predicted performance of a rotating modulation collimator for locating celestial hard x-ray sources," Space Sci. Rev. 8, 534-542 (1968).

12. M. J. Murphy, "The virtues of positive-definite reconstruction of x-ray and gamma-ray images," Nucl. Instrum. Methods A 290, 551-558 (1990).

13. S. Tsuneta, "Image processing software system of the x-ray telescope aboard the Hinotori spacecraft," Ann. Tokyo Astron. Observatory 20, 1-50 (1984).

14. L. N. Mertz, G. H. Nakano, and J. R. Kilner, "Rotational aperture synthesis for x-rays,” J. Opt. Soc. Am. A 3, 2167-2170 (1986).

15. J. R. Kilner and G. H. Nakano, "Design studies for x-ray and gamma ray rotation modulation collimators," in EUV, X-ray and Gamma-Ray Instrumentation for Astronomy and Atomic Physics, C. J. Hailey, ed., Proc. SPIE 1159, 27-33 (1989).

16. J. A. Högbom, "Aperture synthesis with a non-regular distribution of interferometer baselines," Astron. Astrophys. Suppl. Ser. 15, 417-426 (1974).

17. S. J. Wernecke and L. R. D'Addario, "Maximum entropy image reconstruction,” IEEE Trans. Comput. C 26, 351-364 (1977).

18. B. Frieden, "Statistical models for the image restoration problem," Comput. Graphics Image Process. 12, 40-59 (1980).

19. C. A. Lindsey, "Effects of diffraction in multiple-grid telescopes for x-ray astronomy,” J. Opt. Soc. Am. 68, 1708-1715 (1978).

20. E. Bar-Ziv, "Effect of diffraction on the moiré image. I. Theory," J. Opt. Soc. Am. A 2, 371-379 (1985). 